Cahiers de la recherche sur les droits

Cahiers

Fur les Droits fondamentaux

$9 \mid 2011$

Conseil constitutionnel et droits fondamentaux

\title{
Le statut juridique des personnes prostituées en
}

\section{France}

Juliette Lecame

\section{(2) OpenEdition}

\section{Journals}

Édition électronique

URL : https://journals.openedition.org/crdf/5445

DOI : $10.4000 /$ crdf.5445

ISSN : 2264-1246

Éditeur

Presses universitaires de Caen

\section{Édition imprimée}

Date de publication : 1 décembre 2011

Pagination : 103-116

ISBN : 978-2-84133-396-7

ISSN : $1634-8842$

\section{Référence électronique}

Juliette Lecame, "Le statut juridique des personnes prostituées en France », Cahiers de la recherche sur les droits fondamentaux [En ligne], 9 | 2011, mis en ligne le 01 décembre 2012, consulté le 14

novembre 2022. URL : http://journals.openedition.org/crdf/5445; DOI : https://doi.org/10.4000/crdf 5445 


\title{
Le statut juridique des personnes prostituées en France
}

\author{
Juliette LECAME \\ Doctorante contractuelle à I'Université de Caen Basse-Normandie \\ Centre de recherche sur les droits fondamentaux et les évolutions du droit (CRDFED)
}

I. Les contradictions apparentes de la législation française
A. La licéité de l'activité prostitutionnelle
1. La prostitution, une activité tolérée
2. Une activité nécessairement limitée par l'impératif de sauvegarde de l'ordre public

B. L'interdiction détournée et généralisée de la prostitution

II. L'hypocrisie du système: de la protection à la seule criminalisation des prostitués
A. L'indignité de l'activité prostitutionnelle
1. Un système prohibitionniste en puissance
2. Le principe d'indisponibilité du corps humain opposé aux prostitués

B. Les déficiences d'une législation attentatoire aux droits des prostitués

Le phénomène prostitutionnel est traditionnellement abordé sous l'angle du contentieux de la traite des êtres humains et de l'exploitation sexuelle ${ }^{1}$. La réification d'êtres humains à des fins mercantiles est fermement condamnée par de nombreux instruments internationaux, parmi lesquels la Convention de $2005 \mathrm{du}$ Conseil de l'Europe relative à la lutte contre la traite des êtres humains (art. 4), la Charte des droits fondamentaux de l'Union européenne (art. 5-3), ou encore le protocole additionnel à la Convention des Nations unies contre la criminalité organisée de 200o. Afin de lutter au mieux contre ce phénomène attentatoire aux valeurs d'une société démocratique, les
États parties à ces différents textes se doivent notamment d'adopter un dispositif pénal adéquat. Le droit français sanctionne ainsi lourdement l'exploitation prostitutionnelle d'autrui considérée comme «incompatible avec la dignité et la valeur de la personne humaine $»^{2}$. Dénoncés dans les articles 225-4-1 et 225-5 du Code pénal, la traite des êtres humains et le proxénétisme de contrainte et de profit sont punis de 7 ans d'emprisonnement et de 150000 euros d'amende ${ }^{3}$.

Mais au-delà de cette forme moderne d'esclavage, il existe une prostitution volontaire ou consentie, «définie comme la prostitution exercée par les personnes de

1. The Trafficking in Persons Report of Department of State - United States of America, publié en juin 2010, affirme que la traite des êtres humains aurait concerné environ 12,3 millions d'individus en 2009, dont 18 ooo en France (p. 149-151, disponible sur https://www.state.gov/documents/ organization/142979.pdf).

2. Préambule de la Convention des Nations unies pour la répression de la traite des êtres humains et de l'exploitation de la prostitution d'autrui, approuvée par l'Assemblée générale dans sa résolution 317 (IV) du 2 décembre 1949.

3. Pour un exposé plus précis sur la lutte contre la traite et l'exploitation sexuelle, voir A. Cazals, Prostitution et proxénétisme en Europe, Paris, La Documentation française, 1995; A. Weyembergh, "L’Union européenne et la lutte contre la traite des êtres humains», Cahiers de droit européen, $\mathrm{n}^{\mathrm{0}}$ 1-2, 2000, p. 215-251. 
plus de 18 ans qui ont choisi cette activité comme moyen de gagner leur vie» ${ }^{4}$; les textes distinguent le fait d'être prostitué - par quelqu'un d'autre - du fait de se prostituer. À la différence de la condamnation unanime de l'exploitation sexuelle, on observe l'absence de consensus en ce qui concerne les échanges économico-sexuels librement consentis. Les États appliquent à cette activité et à ses acteurs un régime juridique différent, selon qu'ils adoptent le système prohibitionniste (interdiction des échanges économico-sexuels), abolitionniste (tolérance de la prostitution), ou réglementariste (institutionnalisation de l'amour vénal).

Depuis son adhésion à la Convention des Nations unies pour la répression de la traite des êtres humains et de l'exploitation de la prostitution d'autrui du 2 décembre 1949, la France s'est engagée dans la voie de l'abolitionnisme ${ }^{5}$. Ce terme ne doit pas s'entendre comme l'interdiction du phénomène prostitutionnel, mais comme la dénonciation de l'inscription des prostitués dans des registres spéciaux qui empêchent leur reclassement en les marquant du «stigmate de [la] putain ${ }^{6}$. Présenté comme un intermédiaire entre prohibitionnisme et réglementarisme, le système abolitionniste cherche à éradiquer la pratique d'échanges économico-sexuels, mais sans les interdire expressément. L'exercice de la prostitution consentie est toléré afin que les individus qui s'y livrent ne soient pas perçus comme des délinquants. En revanche, toute forme de proxénétisme de profit et de contrainte est sévèrement réprimée, et les autorités doivent mettre en place des politiques sociales à long terme permettant la protection et la réhabilitation progressive des personnes monnayant leurs charmes.

Cet engagement explique qu'aucune disposition de notre droit ne s'intéresse en tant que telle à la prostitution, activité ni illicite ni réglementée. En revanche, pour l'application des articles 225-5 à 225-10 du Code pénal correctionnalisant les comportements proxénètes, il a été nécessaire de définir précisément l'activité sexuelle rétribuée. Selon la chambre criminelle de la Cour de cassation, «la prostitution consiste à se prêter, moyennant rémunération, à des contacts physiques de quelque nature qu'ils soient, afin de satisfaire les besoins sexuels d'autrui $»^{7}$.
La seule réalisation tarifée d'actes de nature sexuelle n'est pas répréhensible. De prime abord, on semble reconnaître aux individus la possibilité de disposer de leur corps et d'offrir contre rémunération des services sexuels, sous réserve de ne pas troubler l'ordre public et la tranquillité d'autrui. Ce raisonnement tend à rejoindre la philosophie réglementariste inspirant les législations allemande, hellénique et néerlandaise ${ }^{8}$. Initialement, ce système se fonde sur le postulat que la prostitution est un phénomène inéluctable et qu'elle est même nécessaire au maintien de l'ordre, puisqu'elle diminuerait les risques de viols ${ }^{9}$. Les autorités encadrent cependant son exercice, notamment par l'instauration de maisons closes et de contrôles sanitaires périodiques, afin de lutter contre l'expansion de maladies vénériennes. Désormais, dans les États réglementaristes, les pratiques sexuelles rétribuées sont expressément légalisées et rattachées à l'exercice de la liberté personnelle.

Distincte de la liberté individuelle qui s'entend du droit à la sûreté garanti par l'article 66 de la Constitution, la liberté personnelle reconnaît aux individus le droit de mener leur vie comme ils l'entendent ${ }^{10}$, dans le respect des droits et libertés d'autrui conformément à l'article 4 de la Déclaration des Droits de l'homme et du citoyen. Il existe ainsi une sphère d'autonomie protégée de l'ingérence des autorités publiques, à l'intérieur de laquelle prime le libre arbitre de l'individu. Selon la pensée réglementariste, la personne qui consent à monnayer ses faveurs est libre de le faire et se voit d'ailleurs octroyer le statut légal de travailleur du sexe. La professionnalisation de la prostitution volontaire est liée à une vision purement mercantile de cette activité singulière, vision retenue par la Cour de justice des communautés européennes dans un arrêt de $2001^{11}$. Loin de toute considération morale, les juges communautaires assimilent l'amour vénal à une activité économique, dès lors que la prestation de service sexuel est réalisée de façon indépendante et que son auteur perçoit l’intégralité de la rémunération.

Si le statut licite de la prostitution en France tend à rapprocher les pensées réglementariste et abolitionniste, on ne peut que constater l'écart insurmontable entre les deux, puisque la seconde souhaite aboutir à la disparition de toute forme d'échange économico-sexuel tarifé. C'est

4. Assemblée parlementaire du Conseil de l'Europe, Prostitution - quelle attitude adopter?, Résolution 1579 (2007), 4 octobre $2007, \$ 4$.

5. L'engagement de la France se fait en plusieurs temps: la loi nº 46-685 du 13 avril 1946 (dite «Marthe Richard») tendant à la fermeture des maisons de tolérance et au renforcement de la lutte contre le proxénétisme interdit les maisons closes et l'ordonnance $\mathrm{n}^{\circ} 60-1245$ du $25 \mathrm{novembre} 1960$ relative à la lutte contre le proxénétisme supprime le fichier national sanitaire et social de la prostitution. La France a ensuite ratifié la Convention onusienne de 1949 le 28 juillet 1960.

6. G. Pheterson, «Le stigmate de putain: déshonneur féminin et indignité masculine», Prisme de la prostitution, Paris, L'Harmattan, 2003, p. 57-94.

7. Cass. crim., nº 95-82.016, 27 mars 1996, Bull. crim., $\mathrm{n}^{\circ} 138$; Droit pénal, 1996, 182, obs. Véron.

8. Concernant les différentes législations européennes en matière de prostitution, voir D. Derycke, Les politiques publiques et la prostitution. Rapport d'information sur l'activité de la délégation aux droits des femmes et à l'égalité des chances entre les hommes et les femmes pour l'année 2000 , 31 janvier 2001, p. 30 (disponible sur http://www.senat.fr/rap/roo-209/roo-209.html).

9. Sur les fondements de la philosophie réglementariste, voir V. Guienne, «La prostitution, une catégorie sociale construite», in Action publique et Prostitution, J. Danet et V. Guienne (dir.), Rennes, Presses universitaires de Rennes, 2006, p. 21-23.

10. CC, déc. $\mathrm{n}^{\circ} 2003-484 \mathrm{DC}$ du 20 novembre 2003, Loi relative à la maîtrise de l'immigration, au séjour des étrangers en France et à la nationalité, Rec., p. 438, cons. 94 .

11. CJCE, 20 novembre 2001, affaire C-286/99, Aldona Malgorzata Jany et autres c. Pays-Bas, Rec., p. 8615; S. Retterer, «L'activité de prostitution exercée à titre indépendant: une activité économique au sens du droit communautaire», Recueil Dalloz, n 27, 2002, p. $2144-2148$. 
d'ailleurs à ce titre que la Convention onusienne de 1949 interdit la reconnaissance et, a fortiori, l'institutionnalisation et la favorisation des rencontres prostitutionnelles. Elle met en revanche l'accent sur la nécessité de favoriser le reclassement des prostitués et prône la mise en œuvre d'un important volet social. Il faut ici souligner que le législateur national s'écarte de la philosophie abolitionniste traditionnelle, puisque, notamment depuis la loi pour la sécurité intérieure de $2003^{12}$, il concentre son action sur l'encadrement strict des manifestations extérieures de l'amour vénal. La multiplication des infractions pénales connexes à l'activité prostitutionnelle fait ainsi muter le système abolitionniste français pour le rapprocher du système prohibitionniste.

Le Danemark, la Norvège, la Chine ainsi que les ÉtatsUnis ${ }^{13}$ interdisent tout échange économico-sexuel tarifé sur le fondement du principe de dignité humaine. Ces États apparentent la prostitution à une violence et un mal absolus qui nient l'humanité de chaque individu. Pareille analyse n'est pas sans rappeler celle suivie par le juge administratif dans l'affaire dite du «lancer de nains». Le 25 octobre 1991, le maire de la commune de Morsangsur-Orge édictait un arrêté municipal interdisant un spectacle de «lancer de nains» dans une discothèque, estimant qu'un tel événement portait atteinte à l'ordre public. Saisi de ce contentieux, le Conseil d'État confirme le 27 octobre 1995 la légalité de l’arrêté municipal en alléguant que «le respect de la dignité de la personne humaine est une composante de l'ordre public [et que] l'autorité investie du pouvoir de police municipale peut, même en l'absence de circonstances locales particulières, interdire une attraction qui porte atteinte à la dignité de la personne humaine ${ }^{14}$.

En plus d'élargir les pouvoirs de police administrative du maire, cette solution affirme que la réification du corps humain, même lorsqu'elle est le fait du protagoniste, doit être interdite sur le fondement d'une conception objectivée de la dignité. Il ne s'agit plus d'une dignité dont le respect est opposé aux tiers, mais directement aux intéressés.

Le système prohibitionniste considère que se prostituer est un acte de chosification de la personne. En échange d'une rémunération, le prostitué permet d'être considéré comme un simple objet sexuel et nie son statut d'être humain. Il faut donc interdire stricto sensu l'activité prostitutionnelle et valoriser le principe d'indisponibilité du corps humain. En ce sens, le droit national tend à se rapprocher du prohibitionnisme, puisque les articles 16-5 et suivants du Code civil posent le principe de non-patrimonialité du corps. Cependant, il existe une différence majeure entre les deux systèmes : l'échange économico-sexuel n'est pas pénalisé en France et il est donc possible de s'y prostituer.

Tel que mis en œuvre en France, le système abolitionniste participe en réalité d'une logique inaboutie: soit l'on reconnaît aux prostitués la liberté de marchander leurs faveurs et donc de disposer de leur corps, soit la volonté d'éradiquer tout phénomène prostitutionnel contraire à la dignité et subjective et objective des individus s'y livrant se présente comme un obstacle insurmontable à la liberté personnelle. Il semble impossible de concilier ces deux logiques fondamentalement antagonistes. Le régime flou auquel les personnes prostituées sont soumises apparaît nécessairement attentatoire à leurs droits et libertés. Si les autorités publiques reconnaissent l'indignité des relations sexuelles tarifées, il faut obligatoirement prohiber expressément ce phénomène afin de protéger et d'assurer le respect de la dignité et de l'humanité des prostitués. En revanche, si la prostitution licite découle de la liberté personnelle de ses acteurs, il est indispensable qu'aucune atteinte arbitraire n'y soit portée et que le libre exercice prostitutionnel soit protégé.

Du fait de cet «entre-deux», de ce «non-statut» officiel de la prostitution, la législation apparaît nécessairement insatisfaisante. L'encadrement toujours plus strict des modalités d'exercice de l'activité licite de prostitution aboutit à interdire en pratique les échanges économicosexuels tarifés, et surtout à en stigmatiser et marginaliser les auteurs. Les prostitués, martyrs à sauver de leur condition, sont désormais des délinquants coupables de racolage et de troubles à l'ordre public. Non seulement contradictoire (I), le système français se révèle d'une grande hypocrisie en ce qu'il glisse de l'impératif de protection des personnes prostituées à leur seule criminalisation (II).

\section{Les contradictions apparentes de la législation française}

En ratifiant le 28 juillet 1960 la Convention abolitionniste des Nations unies, la France a nécessairement dû faire évoluer sa législation en matière de prostitution consentie. L'acte prostitutionnel ne peut plus être ni réglementé ni pénalisé. L'affirmation de la licéité de l'activité prostitutionnelle (A) n'est pas sans soulever des interrogations majeures, notamment au regard du paradoxe de la législation actuelle: l'échange économico-sexuel est admis, mais il existe une interdiction détournée et généralisée du phénomène prostitutionnel, portée par diverses infractions pénales (B).

\section{A. La licéité de l'activité prostitutionnelle}

La reconnaissance du caractère licite des échanges économico-sexuels ne doit pas s'entendre comme la reconnaissance d'une véritable liberté de monnayer ses faveurs, mais comme la tolérance de la prostitution (1), tolérance mise à mal par l'impératif de sauvegarde de l'ordre public (2).

12. Loi n ${ }^{2} 2003-239$ du 18 mars 2003 pour la sécurité intérieure, publiée au JORF du 19 mars 2003, p. 4761 sq.

13. À l'exception de l'Arizona, du Nevada et de l'Oregon.

14. CE, Ass. plén., 27 octobre 1995, $\mathrm{n}^{\circ}$ 136727, Commune de Morsang-sur-Orge et ville d'Aix-en-Provence, Recueil Lebon, 372 , cons. 2 et 3 


\section{La prostitution, une activité tolérée}

L'absence de dénonciation formelle de l'amour vénal découle de la neutralité prônée par le système abolitionniste. À la faveur de l'article 5 de la Déclaration de 1789 qui dispose que «tout ce qui n'est pas défendu par la loi ne peut être empêché», il serait tentant de supposer l'existence d'une liberté de se prostituer en France, à l'instar de celle consacrée dans les États réglementaristes.

Les législations reconnaissant la liberté de se livrer à la prostitution sont fondées sur un raisonnement éminemment individualiste, récemment valorisé par la Cour européenne (CEDH) qui a opéré une relecture approfondie du texte de la Convention de 1950 "à la lumière des conditions d'aujourd'hui » ${ }^{15}$. La compréhension du droit au respect à la vie privée et familiale consacré par l'article 8 de la $\mathrm{CEDH}$ a ainsi évolué au fil des années pour dépasser le simple cadre d'une protection de l'inviolabilité du domicile et des correspondances personnelles contre les immixtions des autorités publiques. Au moyen d'une conception rénovée de la vie privée, on reconnaît aux individus une autonomie personnelle de plus en plus étendue, notamment en ce qui concerne la relation qu'ils entretiennent avec leur propre corps. La liberté sexuelle, qui échappe pourtant à toute consécration textuelle, est ainsi incluse dans le champ de l'article 8 de la CEDH dès $1985^{16}$ et s'affirme progressivement comme une dimension fondamentale de l'autonomie personnelle, «à mesure que le poids de la morale traditionnelle s'affaiblit ${ }^{17}$.

Toutes les manifestations de la sexualité consentie sont intégrées progressivement dans le droit au respect de la vie privée, y compris les plus violentes. Dans l'affaire K.A. et A.D. c. Belgique, la Cour affirme que «le droit d'entretenir des relations sexuelles découle du droit de disposer de son corps, partie intégrante de la notion d'autonomie personnelle ${ }^{18}$. Confrontés à des actes sadomasochistes d'une grande violence, les juges opèrent un revirement de jurisprudence - vivement dénoncé par la doctrine - et décident « que le droit pénal ne peut, en principe, intervenir dans le domaine des pratiques sexuelles consenties qui relèvent du libre arbitre des individus ». Ils redonnent ici toute sa force au consentement de la personne humaine en reconnaissant le droit de libre disposition de son corps.

La protection offerte à l'épanouissement personnel de l'individu apparaît illimitée, puisque la notion de «vie privée » englobe également le droit de nouer et de développer avec autrui des relations personnelles, sociales et économiques. Il n'y a «aucune raison de principe de considérer cette notion comme excluant les activités professionnelles ou commerciales ${ }^{19}$. Dès lors, la combinaison entre le principe d'autodétermination sexuelle et celui d'inclusion de la vie professionnelle dans le champ de l'article 8 de la Convention permet aux États réglementaristes de reconnaître une liberté de se prostituer, protégée en tant que telle ${ }^{20}$. La consécration de cette liberté va autoriser la professionnalisation de l'activité prostitutionnelle, dont les vitrines du «quartier rouge» d'Amsterdam sont l'exemple le plus célèbre. Dès lors qu'ils sont consentants, les individus monnayant leurs charmes bénéficient du statut légal de "travailleur du sexe», à condition toutefois de respecter les droits et libertés des tiers ${ }^{21}$.

L'on ne peut que constater l'impossibilité d'une telle situation en France. L'affirmation de la licéité de l'activité prostitutionnelle ne saurait s'apparenter à la reconnaissance d'une liberté de se prostituer ressortissant du champ de la liberté personnelle. La philosophie abolitionniste aboutit certes à l'indifférence du droit pénal à l'égard des échanges économico-sexuels librement consentis, mais elle refuse avec force leur institutionnalisation. Il ne faut pas incriminer les personnes qui marchandent leurs faveurs, mais il ne faut pas non plus favoriser leur action. Cette volonté est relayée par le principe de non-patrimonialité du corps humain ${ }^{22}$ ainsi que par les articles 6, 1131 et 1133 du Code civil qui rappellent la nullité de tout contrat fondé sur une cause illicite ou contraire aux bonnes mœurs, tel le contrat de stupre ${ }^{23}$.

La tolérance souhaitée par la Convention onusienne de 1949 ne s'entend finalement que du seul point de vue pénal. Le statut licite de la prostitution rappelle que les personnes s'y livrant doivent être considérées comme des

15. CEDH, 25 avril 1978, $\mathrm{n}^{\circ}$ 5856/72, Tyrer c. Royaume-Uni, série A, $\mathrm{n}^{\circ} 26, \$ 31$.

16. CEDH, 26 mars $1985, \mathrm{n}^{\circ} 8978 / 80$, X. et Y. c. Pays-Bas, série A, $\mathrm{n}^{\circ} 91, \$ 22:$ «La “vie privée" recouvre l'intégrité physique et morale de la personne et comprend la vie sexuelle».

17. D. Borrillo et D. Lochak, La Liberté sexuelle, Paris, PUF, 2005, p. 1.

18. CEDH, 27 février 2005, $\mathrm{n}^{\circ} 42758 / 98$ et 45558/99, K.A. et A.D. c. Belgique, $\$ 82-83$; J.-P. Marguénaud, «Sadomasochisme et autonomie personnelle», Revue trimestrielle de droit civil, 2005, p. 341-343; J.-P. Marguénaud, «Liberté sexuelle et droit de disposer de son corps", Droits. Revue française de théorie, de philosophie et de culture juridique, $\mathrm{n}^{\circ} 49,2009$, p. 19-27.

19. CEDH, 16 décembre 1992, $\mathrm{n}^{\circ}$ 13710/88, Niemietz c. Allemagne, série A, nº 251-B, $\$ 29$. Solution réaffirmée dans l'arrêt Sidabras et Dziautas c. Lituanie, 27 juillet 2004, $\mathrm{n}^{\circ} 55480 / 00$ et 59330/o0, Rec. 2004-VIII, sur le fondement de l'article $1 \$ 2$ de la Charte sociale européenne qui pose le droit de gagner sa vie par un travail librement entrepris.

20. Il est important de souligner que ce sont les États réglementaristes qui mettent en exergue la jurisprudence de la CEDH pour justifier leur législation. On ne peut en revanche pas être certain que la Cour suive la même analyse en la matière. En raison de l'absence de consensus européen, elle se refuse à prendre une position de principe sur les différentes législations en vigueur relatives à la prostitution volontaire.

21. L'article $8 \$_{2}$ de la CEDH autorise une ingérence des pouvoirs publics dans le domaine de la sexualité lorsqu'il existe des raisons particulièrement graves d'atteinte à la santé ou à la morale d'autrui. La reconnaissance de la liberté de se prostituer ne saurait aboutir à heurter ou blesser brutalement «la décence sexuelle des personnes douées d'une sensibilité normale» (CEDH, 24 mai 1988, ${ }^{\circ}$ 10737/84, Müller et autres c. Suisse, $\$ 36$; sur ce point, voir J.-M. Larralde, «Libre disposition de son corps et préférences sexuelles», in La Libre Disposition de son corps, J.-M. Larralde (dir.), Bruxelles, Bruylant, 2009, p. 282-283).

22. Voir infra.

23. CA Colmar, 19 janvier 1958, Dalloz, 1958, p. 163. 
individus lambda, titulaires des mêmes droits et libertés. Néanmoins, en raison du caractère singulier des manifestations de l'amour vénal dans l'espace public, leurs libertés individuelles peuvent être restreintes sur le fondement de l'impératif de sauvegarde de l'ordre public.

\section{Une activité nécessairement limitée par l'impératif de sauvegarde de l'ordre public}

Reconnu comme objectif à valeur constitutionnelle ${ }^{24}$, le principe de sauvegarde de l'ordre public est synthétisé à l'article L. 2212-2 du Code général des collectivités territoriales: «La police municipale a pour objet d'assurer le bon ordre, la sûreté, la sécurité et la salubrité publiques ». La prostitution, activité licite, n'est pas constitutive en elle-même d'un trouble à l'ordre public; en revanche, les nuisances "périphériques» qu'elle peut générer le sont. Ainsi, les rassemblements de prostitués et de clients, cause de nuisances sonores ou de rixes, les stationnements impromptus sur la voie publique qui gênent la circulation ou encore la découverte de préservatifs usagers ou de déchets en tout genre peuvent justifier l'action du maire.

Les manifestations extérieures et dérangeantes de l'amour vénal doivent non seulement être conciliées avec «l'ordre public matériel et extérieur ${ }^{25}$, mais également avec l'exigence de moralité publique, nouvelle composante de l'ordre public. Cette notion ne connaît pas de définition exacte, si ce n'est qu'elle représente «le minimum d'idées morales communément admises à un moment donné par la moyenne des citoyens ${ }^{26}$. À la différence de l'ordre public matériel qui se rattache à des faits concrets et identifiables, la protection de la moralité publique se révèle nécessairement plus vague en raison de l'évolution des mœurs. Néanmoins, le contentieux a été objectivé dès $1959^{27}$, puisque le titulaire de l'autorité de police doit préciser en quoi le contexte local fait que l'ordre public risque d'être troublé. Il ne peut pas se référer à la seule immoralité d'une activité; il doit justifier de circonstances locales particulières. Ainsi, un arrêté municipal «antiprostitution » est légitimement édicté afin d'empêcher que l'activité prostitutionnelle ne s'exerce près d'un lieu de culte ${ }^{28}$ ou à proximité d'établissements d'enseignement ou de lieux fréquentés par des enfants ${ }^{29}$.
En revanche, la mesure de police ne peut pas interdire de façon absolue et généralisée la présence et les allées et venues des personnes prostituées. Cette exigence résulte de l'arrêt Benjamin du 19 mai $1933^{30}$, dans lequel le Conseil d'État affirme la nécessité de concilier au mieux l'impératif de maintien de l'ordre public et le respect de la liberté en cause. Lorsqu'il contrôle la légalité d'une mesure de police générale, le juge administratif s'assure que celle-ci répond de façon adéquate aux risques de trouble à l'ordre public. Il exerce un contrôle maximum aussi bien sur la motivation que sur les prescriptions de l'acte. La seule potentialité d'une menace de désordre n'est pas de nature à justifier la restriction d'une liberté fondamentale; il faut que le risque soit précis et avéré par des plaintes de riverains ou de tiers. De plus, l'autorité de police ne peut prendre que des mesures strictement nécessaires et proportionnées aux risques de trouble à l'ordre public. L'importance de l'atteinte à la liberté d'aller et venir des acteurs prostitutionnels doit être la seule réponse à la gravité du désordre encouru.

Une circulaire du ministère de l'Intérieur datée du 23 août 2002 a rappelé cette exigence, suite à divers arrêtés municipaux « anti-prostitution » attentant excessivement aux libertés des individus ${ }^{31}$. Les seules restrictions légitimes sont celles limitées dans le temps et l'espace. Ainsi, la décision réglementaire qui prohibe l'activité de prostitution sur l'ensemble d'une commune - à l'exception de six portions de trottoirs - de $21 \mathrm{~h} 30$ à 6 heures du matin est annulée par le juge administratif au motif qu'elle consiste «en une interdiction globale de la prostitution chaque nuit sur la quasi-totalité du territoire ${ }^{32}$. En revanche, la limitation ponctuelle de la liberté de déplacement et de stationnement sur la voie publique des personnes monnayant leurs faveurs dans des lieux précis s'apparente à une mesure proportionnée et nécessaire au maintien de l'ordre public ${ }^{33}$. Par le biais de son contrôle maximum, le juge administratif se pose comme le garant des libertés reconnues aux prostitués.

Malgré cela, il reste à s'interroger sur l'opportunité de cette réglementation administrative des échanges économico-sexuels, en raison de la redondance entre les mesures de police municipale et certaines infractions pénales, telles que les délits de racolage public ${ }^{34}$ et d'exhibition sexuelle ${ }^{35}$

24. CC, déc. $\mathrm{n}^{\circ}$ 82-141 DC du 27 juillet 1982, Loi sur la communication audiovisuelle, Rec., p. 48, cons. 5 .

25. M. Hauriou, Précis de droit administratif, $11^{\mathrm{e}}$ édition, Paris, Sirey, 1927, p. 471.

26. Définition du commissaire du gouvernement Guldner dans ses conclusions sur l'arrêt du CE, 20 décembre 1957, Société nationale d'édition cinématographique, Sirey, $\mathrm{n}^{\circ} 3,1958$, p. 73 .

27. CE section, 18 décembre 1959, $\mathrm{n}^{\circ} 36835$ 36428, Société Les films Lutétia, Recueil Lebon, p. 623, Sirey, ${ }^{\circ}$ 9, 1960, concl. Mayras, cons. 2 in fine.

28. CE, 11 mai 1977, $\mathrm{n}^{\circ}$ 01567, Ville de Lyon, Recueil Lebon, 235.

29. Cass. crim., 9 mai 1961. Voir également CE, 8 juin 2005, $\mathrm{n}^{\circ} 281084$, Commune de Houilles à propos de l'interdiction d'un sexe-shop près d'établissements scolaires et d'installations destinées à la jeunesse.

30. CE, 19 mai 1933, $\mathrm{n}^{\circ} 1741317520$, Benjamin, cons. 3 .

31. Ministre de l'Intérieur, de la Sécurité intérieure et des Libertés locales, Circulaire concernant les arrêtés municipaux relatifs à la prostitution sur la voie publique, NOR/INT/D/o2/00165/C, 23 août 2002.

32. Jugement du TA de Caen, 18 novembre 2003, $\mathrm{n}^{\circ}$ 03-462, Préfet du Calvados, cons. 5 in fine.

33. Cass. crim., 9 mai 1961 à propos de l'interdiction de l'activité prostitutionnelle aux abords d'un établissement scolaire entre 11 heures et $12 \mathrm{~h} 30$, et entre $13 \mathrm{~h} 30$ et 22 heures.

34. Code pénal, art. 225-10-1 (voir infra).

35. Code pénal, art. R. 623-1. 
ou les contraventions pour tapage nocturne ${ }^{36}$ ou stationnement dangereux ${ }^{37}$. À cet égard, la circulaire de 2002 rappelle que «l'existence de possibilités de poursuites pénales, notamment en raison de la difficulté de rapporter la preuve des faits incriminés, ne doit pas conduire à considérer que l'arrêté du maire n'est pas nécessaire ». Néanmoins, l'ensemble de ces mesures semble davantage révélateur d'une politique d'hygiénisme social qui conduit à l'encadrement en amont et en aval de la prostitution et, finalement, à son interdiction détournée et généralisée.

\section{B. L'interdiction détournée et généralisée de la prostitution}

Selon les articles 1 et 2 de la Convention onusienne de 1949, seuls les proxénètes qui exploitent et favorisent la prostitution doivent être punis; la répression des personnes prostituées en raison de leur activité n'est pas évoquée. De même, dans une résolution de 2007 relative à l'attitude à adopter face à la prostitution, le Conseil de l'Europe recommande aux États parties «d'éviter les normes et politiques discriminatoires qui poussent les prostitué(e) $s$ à la clandestinité [...], notamment en s'abstenant d'ériger en infractions pénales [leurs] actions ${ }^{38}$. Cependant, le législateur national affirme le caractère délictueux du racolage public, jugeant l'infraction nécessaire pour combattre les réseaux de prostitution forcée et pour en protéger les victimes.

Dans sa version actuelle ${ }^{39}$, l'article 225-10-1 du Code pénal dispose que «le fait, par tout moyen, y compris par une attitude même passive, de procéder publiquement au racolage d'autrui en vue de l'inciter à des relations sexuelles en échange d'une rémunération ou d'une promesse de rémunération est puni de 2 mois d'emprisonnement et de 3750 euros d'amende». Se pose dès lors la question de savoir s'il est possible de se prostituer en France, puisque le moyen préparatoire par excellence de cette activité constitue un délit au champ matériel extrêmement étendu. L'incitation publique - c'est-à-dire qui a lieu sur la voie publique, dans un lieu ouvert au public ou portée à la connaissance du public par le biais de petites annonces ${ }^{40}$ - à une pratique sexuelle tarifée est interdite, qu'elle soit sans ambiguité ou déduite d'une certaine attitude racoleuse. Si le délit de racolage actif peut être aisément constaté à l'égard d'une personne qui déambule sur la voie publique et qui met ostensiblement sa poitrine en valeur en s'approchant de passants pour leur parler ${ }^{41}$, l'imprécision de l'élément constitutif de l'infraction de racolage passif est en revanche patente. La qualification de ce délit apparaît problématique dans la mesure où elle a trait uniquement à l'attitude remarquée du prostitué, à sa tenue vestimentaire plus ou moins provocante ou à son immobilité sur la voie publique en l'absence de toute incitation sexuelle explicite de sa part.

Dans sa décision du 13 mars $2003^{42}$, le Conseil constitutionnel valide la constitutionnalité de la disposition, sans répondre aux requérants qui dénonçaient une atteinte au principe constitutionnel de légalité des délits et des peines. Tirée de l'article 8 de la Déclaration des Droits de l'homme et du citoyen, cette règle impose au «législateur de définir les infractions en termes suffisamment clairs et précis pour exclure [l'application] arbitraire ${ }^{43}$ de la loi, afin que soient respectés le droit à la sûreté des individus et le principe d'égalité devant la loi. Fondant l'ensemble de son raisonnement sur les troubles occasionnés par le racolage public et sur la nécessité de lutter contre le proxénétisme et le trafic des êtres humains, le Conseil fait primer les exigences de l'ordre public sur le principe de légalité des délits et des peines, pourtant manifestement bafoué ${ }^{44}$.

Dans quelle mesure la répression d'une sollicitation en vue d'un échange économico-sexuel «par une attitude même passive» répond-elle aux exigences constitutionnelles de clarté et de précision? Il revient en effet au juge de définir et de contrôler a posteriori l'élément matériel du

36. Code de la route, art. R. 417-9 sq.

37. Code pénal, art. 222-32.

38. Assemblée parlementaire du Conseil de l'Europe, Prostitution - Quelle attitude adopter?, $\$ 11.3$ et 11.3.1.

39. Sur l'évolution mouvementée du délit de racolage public, voir M.-H. Renaut, «L'ordre public et la prostitution ou l'Histoire n'est qu'un perpétuel recommencement", Revue de science criminelle et de droit pénal comparé, n 2, 2006, p. 293-305. L'actuel article 225-10-1 du Code pénal a été introduit par la loi pour la sécurité intérieure de 2003

40. Voir Cass. crim., 30 octobre 1956, Bull. crim., $\mathrm{n}^{\circ} 690$.

41. CA Toulouse, $3^{\mathrm{e}}$ chambre correctionnelle, 4 mai 2005, $\mathrm{n}^{\mathrm{o}}$ 2005-271647. Voir également CA Paris, $13^{\mathrm{e}}$ chambre correctionnelle, section A, 29 novembre 2004, $\mathrm{n}^{\mathrm{o}}$ 2004-271968; CA Paris, $13^{\mathrm{e}}$ chambre correctionnelle, section B, 22 septembre 2004, $\mathrm{n}^{\mathrm{o}}$ 2004-254176; CA Paris, $13^{\mathrm{e}}$ chambre correctionnelle, section B, 4 juin 2004, $\mathrm{n}^{\circ}$ 2004-253420.

42. CC, déc. $\mathrm{n}^{\mathrm{0}}$ 2003-467 DC du 13 mars 2003, Loi pour la sécurité intérieure (ci-après, déc. LSI), Rec., p. 211, cons. 58 à 65.

43. CC, déc. $\mathrm{n}^{\circ}$ 80-127 DC du 20 janvier 1981, Loi renforçant la sécurité et protégeant la liberté des personnes, Rec., p. 15, cons. 7.

44. On aurait logiquement attendu que le Conseil relève l'imprécision de l'expression «attitude même passive», comme il l'avait fait dans sa décision n 98-399 DC du 5 mai 1998, Loi relative à l'entrée et au séjour des étrangers en France et au droit d'asile. En l'espèce, il censure une disposition prévoyant que les «associations à but non lucratif à vocation humanitaire» ne peuvent être déclarées responsables pénalement de l’infraction d'aide directe ou indirecte à l'entrée et au séjour irréguliers d'un étranger en France. Le Conseil relève que la notion de "vocation humanitaire » "n'a été précisée par aucune loi [alors pourtant que de sa] reconnaissance peut résulter le bénéfice d['une] immunité pénale» (cons. 5 à 7 ). De la même manière, le délit de racolage passif aurait appelé censure pour violation du principe de légalité des délits et des peines, puisqu'il n'a jamais été défini que par des à-coups jurisprudentiels, parfois contradictoires. À cet égard, la circulaire du 18 janvier 1994 portant commentaire de la partie réglementaire du nouveau Code pénal et des modifications de nature réglementaire nécessitées par son entrée en vigueur (Bulletin officiel du ministère de la Justice, $\mathrm{n}^{\circ}$ 94/53, p. 90-170) précisait d'ailleurs que «la contravention de racolage passif, aujourd'hui prévue par l'article R. $34-13^{\circ}$, n'a pas été conservée en raison de l'imprécision de ses éléments constitutifs». 
délit de racolage passif. Son intervention tardive ne permet pas de garantir le respect de la liberté individuelle de l'individu puisque le placement en garde à vue de celuici sur le fondement de l'article 225-10-1 du Code pénal est décidé par un officier de police judiciaire (OPJ) qui apprécie seul l'attitude «suggestive» du prévenu. L'imprécision des termes du délit peut être la cause de dérives policières et de gardes à vue injustifiées. Ainsi, les juges ont infirmé des interpellations d'individus soupçonnés de racolage passif lorsque la décision de l'OPJ était motivée uniquement par le lieu de stationnement ${ }^{45}$ de la personne ou par son habillement ${ }^{46}$. Ces éléments sont à eux seuls insuffisants à caractériser un comportement racoleur et à démontrer en quoi l'attitude est «en vue d'inciter à des relations sexuelles » contre rémunération.

Les diverses et nombreuses jurisprudences relatives à ce contentieux ne donnent pas de critères fixes et précis permettant d'identifier rigoureusement une pose lascive ou un regard insistant. Ainsi, une femme assise sur le siège conducteur d'une camionnette, vêtue d'un déshabillé rose ouvert et transparent, se rend coupable de racolage passif ${ }^{47}$, à la différence d'un travesti légèrement habillé et stationnant longuement sur un trottoir connu comme lieu de prostitution ${ }^{48}$. Ces solutions contradictoires sont la conséquence directe de l'imprécision de l'incrimination et donc de sa contrariété au principe de légalité des délits et des peines. S'ajoutent à ce risque sérieux d'arbitraire des solutions éminemment contestables, car fondées uniquement sur la connaissance par les policiers de l'activité prostitutionnelle de certains individus. Une présomption de racolage est établie à l'égard de personnes déjà connues des services judiciaires pour se prostituer ${ }^{49}$. Pour différencier une péripatéticienne d'une femme courtvêtue mais sans intention "commerciale», les OPJ basent leur raisonnement sur le passé pénal ou sur la notoriété du prostitué, et non pas sur un élément objectif et extérieur.

Dénoncé sans relâche par les associations ${ }^{50}$, le délit de racolage passif sur la voie publique reflète la volonté du législateur d'empêcher par tout moyen l'exercice de la prostitution, volonté relayée par d'autres incriminations. Si aucune disposition législative ou réglementaire n'interdit la réalisation d'une pratique sexuelle en échange d'une rémunération, l'exercice de l'amour vénal est prohibé en tout lieu. L'interdiction est expresse dans les lieux publics sur le fondement du délit d'exhibition sexuelle ${ }^{51}$. Elle apparaît implicitement en ce qui concerne les véhicules et les espaces privés ouverts au public, puisque ceux qui mettent à la disposition des prostitués ces locaux sont réprimés pour proxénétisme. Ainsi, les articles 225-10 $1^{\circ}$ et $2^{\circ} \mathrm{du}$ Code pénal prohibent la tenue d'établissements de prostitution et le fait que le gérant d'un établissement quelconque ouvert au public accepte ou tolère «qu'une ou plusieurs personnes se livrent à la prostitution à l'intérieur de l'établissement ou de ses annexes ou recherchent des clients en vue de la prostitution ». Si cette infraction se comprend lorsque la commission du délit de racolage est permise par l'attitude du gérant - qui se rend alors complice au sens de l'article 121-7 dudit Code -, elle est plus discutable quand celui-ci ne fait que tolérer la réalisation d'une activité licite. Le fait principal - l'acte prostitutionnel - n'est pas punissable, mais l'aide ou l'assistance, même indirectes, le sont. En raison de l'existence de ces dispositions, les tenanciers d'hôtel doivent refuser la présence de personnes désirant se livrer à la prostitution s'ils ne veulent pas se voir imposer la fermeture définitive ou pour une durée de 5 ans de l'établissement utilisé en vue de la prostitution, ou le retrait définitif de leur licence de débit de boissons ou de restaurant ${ }^{52}$.

Où la prostitution peut-elle donc être exercée? La réponse est sans équivoque: la pratique sexuelle rétribuée ne peut être réalisée nulle part sans heurter une disposition législative. L'article 225-10 $3^{\circ}$ sanctionne l'aide à la prostitution constituée par le fait de vendre ou de tenir à la disposition d'une personne des locaux ou emplacements non utilisés par le public, en sachant qu'elle s'y livrera à la prostitution. A fortiori, cela signifie qu'un individu ne peut pas en principe se prostituer dans un lieu privé, comme un local commercial désaffecté, ou dans un appartement, sauf à cacher ses intentions au vendeur ou au loueur. L'infraction est entendue largement puisqu'elle est constituée dans le cadre aussi bien d'une vente que d'une location ${ }^{53}$ et lorsque le possesseur initial du lieu a connaissance de l'activité soit au moment de la transaction, soit après ${ }^{54}$. Le même subterfuge législatif se retrouve

45. Cass. crim., 28 novembre 1962, Dalloz, 1963, p. 62; CA Toulouse, $3^{\mathrm{e}}$ chambre correctionnelle, 28 juin $2006, \mathrm{n}^{\circ} 2006-308415$

46. Cass. crim., 13 novembre 1963, Bull. crim., $\mathrm{n}^{\circ} 320,1963$; CA Caen, chambre correctionnelle, 21 juin $2004, \mathrm{n}^{\circ} 2005-029291$.

47. CA Paris, $13^{\mathrm{e}}$ chambre correctionnelle, section B, 9 février 2005, $\mathrm{n}^{\circ}$ 2005-274044; CA Reims, chambre correctionnelle, 25 février $2004, \mathrm{n}^{\circ} 2004$ 275508. Les mêmes solutions sont adoptées à propos d'individus portant des tenues vestimentaires moulantes de nature à attirer le regard (CA Paris, $13^{\mathrm{e}}$ chambre correctionnelle, section 4, 3 février 2004, $\mathrm{n}^{\mathrm{o}}$ 2004-291305; CA Toulouse, $3^{\mathrm{e}}$ chambre correctionnelle, 27 juillet 2005, $\mathrm{n}^{\circ} 2005-284530$ ).

48. CA Rouen, 10 mars 2004, JurisClasseur périodique, 2005, IV.1429.

49. CA Amiens, $6^{\mathrm{e}}$ chambre correctionnelle, 7 octobre 2004, $\mathrm{n}^{\circ}$ 2005-275448; CA Rouen, chambre correctionnelle, 7 octobre $2004, \mathrm{n}^{\circ} 2004-272047$; CA Toulouse, $3^{\mathrm{e}}$ chambre correctionnelle, 9 juin $2005, \mathrm{n}^{0} 2005-274982$.

50. Il semble cependant que cette contrariété au principe de légalité des délits et des peines doive perdurer. La disposition litigieuse ayant été déclarée conforme à la Constitution dans les motifs et dispositif par les Sages, aucune question prioritaire de constitutionnalité sur le fondement de l'article 61-1 de la Constitution ne sera recevable en la matière, à moins d'un changement de droit ou de fait.

51. Code pénal, art. 222-32: «L'exhibition sexuelle imposée à la vue d'autrui dans un lieu accessible aux regards du public est punie d'un an d'emprisonnement et de 15 ooo euros d'amende».

52. Code pénal, art. 225-22.

53. CA Paris, 12 février 2001, $\mathrm{n}^{\circ}$ 2001-143673.

54. CA Paris, $11^{\mathrm{e}}$ chambre, 3 juillet 1975, à propos d'un propriétaire qui avait laissé la locataire d'un studio se prostituer alors qu'il avait reçu avis de cette activité de diverses façons. Voir également CA Paris, $11^{\mathrm{e}}$ chambre, 5 novembre 1970, JurisClasseur périodique, édition générale, 1971, II, 16667. En pratique, le propriétaire est informé de l'exercice de la prostitution par le ministère public, lui-même alarmé par les plaintes des voisins et riverains 
à l'article 225-10 $4^{\circ}$ du Code pénal relatif au proxénétisme "voiturier»; on n'interdit pas à un individu d'acquérir une camionnette en vue de se prostituer, mais à un tiers de vendre, de louer ou de mettre à disposition d'une personne «des véhicules de toute nature» en sachant que celle-ci y monnayera ses charmes ${ }^{55}$.

Le système abolitionniste, tel que mis en œuvre en France, se révèle parsemé de contradictions. Si l'acte sexuel tarifé n'est pas en soi constitutif d'un délit pénal, sa réalisation est en pratique impossible. D'un point de vue spatial, l'activité vénale ne peut être exercée ni dans un lieu public ni dans un lieu privé, à l'exception éventuellement du domicile du client. Mais l'infraction volontairement large de racolage public empêche a priori toute rencontre entre les deux protagonistes. Ces mesures sont certes utiles pour s'assurer que la prostitution n'est pas contrainte et que les personnes s'y livrant ne sont pas en danger, mais quid pour celles qui revendiquent leur activité comme librement choisie? La prostitution n'étant pas expressément interdite, rien ne saurait justifier l'incrimination de ceux et celles qui s'y prêtent. Rien, sauf peut-être à considérer qu'une telle activité porte nécessairement atteinte à la dignité de la personne humaine et que, sur ce fondement, il est indispensable de la proscrire.

Plus que contradictoire, le système français apparaît ainsi hypocrite. Sous couvert de la neutralité abolitionniste, le législateur adopte une position proche de celle des États prohibitionnistes. Cependant, il ne va pas jusqu'au bout de sa logique, puisque, au lieu d'aider à la protection effective de la dignité des personnes monnayant leurs charmes, il ne fait que criminaliser celles-ci.

\section{L'hypocrisie du système: de la protection à la seule criminalisation des prostitués}

L'imposture du système est en réalité double. En plus d'adhérer implicitement au précepte prohibitionniste selon lequel l'activité prostitutionnelle est contraire à la dignité de la personne (A), le législateur refuse d'adopter des mesures permettant de lutter contre l'inhumanité du phénomène et de l'éradiquer. Son action se révèle déficiente et particulièrement attentatoire aux droits des personnes prostituées (B).

\section{A. L'indignité de l'activité prostitutionnelle}

L'adoption «cachée» de la philosophie prohibitionniste (1) conduit à opposer aux personnes prostituées le principe de l'indisponibilité de leur corps (2).

\section{Un système prohibitionniste en puissance}

Le lien inextricable entre prostitution et dignité de la personne humaine est affirmé dès le préambule de la Convention abolitionniste du 2 décembre 1949 qui relève l'incompatibilité de la prostitution et du mal qui l'accompagne - la traite des êtres humains aux fins d'exploitation sexuelle - avec le principe de dignité. Le droit interne n'est pas en reste puisque les diverses infractions de proxénétisme et de racolage public sont, depuis la réforme de 1992, contenues dans le chapitre relatif aux «atteintes à la dignité de la personne». Les parlementaires affirment, quant à eux, que «la prostitution n'est ni un métier, ni un mal nécessaire. C'est une atteinte à la dignité humaine, une violence ${ }^{56}$. Cependant, il n'est pas certain que dans ces différentes hypothèses, le terme de dignité renvoie à la même acception.

Dans sa décision Bioéthique du 27 juillet $1994^{57}$, le Conseil constitutionnel précise le contenu et la portée de cette notion, encore largement indéterminée. Le principe constitutionnel de sauvegarde de la personne humaine est en lien direct avec ceux d'inviolabilité et d'intégrité du corps humain. Le Conseil affirme avec force que l'individu ne doit pas être avili ou asservi, mais traité avec l'humanité qui lui est due. C'est à ce titre que l'on peut condamner la prostitution forcée, puisque contraindre une personne à l'exploitation sexuelle s'assimile à organiser son viol, crime constitué par «tout acte de pénétration sexuelle, de quelque nature qu'il soit, commis sur la personne d'autrui par violence, contrainte, menace ou surprise ${ }^{58}$. Afin que l'intégrité physique et l'inviolabilité du corps humain soient respectées, l'État doit adopter une législation nationale à même de réprimer de telles violences. La protection de la dignité subjective des personnes prostituées passe ainsi nécessairement par l'établissement des délits de proxénétisme de contrainte, de menace ainsi que de profit, quand le souteneur oblige la victime à continuer de monnayer ses charmes afin d'en retirer un bénéfice personnel. En revanche, la théorie du consentement implique a contrario qu'une relation sexuelle désirée ne peut en principe relever du droit pénal. Dès lors, comment comprendre que le fait d'aider ou d'assister une personne qui se prostitue volontairement et de façon indépendante relève de l'article 225-5 du Code pénal?

Le fondement de la philosophie abolitionniste réside dans la volonté de garantir la dignité subjective des personnes prostituées exploitées et violentées par autrui. La France dépasse nécessairement ce cadre puisqu'elle ne se contente pas de réprimer l'exploitation sexuelle. Elle considère également comme contraire à la dignité d'un individu le fait de le tolérer dans un lieu ouvert au public,

55. C'est précisément cette nuance qui a permis au Conseil constitutionnel, dans sa décision LSI, de valider cette disposition en considérant, d'une part, que le droit de propriété des prostitués était sauf et, d'autre part, que la disposition ne portait pas atteinte «à la liberté d'entreprendre des vendeurs qui trouve sa limite dans le fait de contribuer, en toute connaissance de cause, à des activités illicites ou contraires à l'ordre public» (cons. 67).

56. D. Derycke, Les politiques publiques et la prostitution..., p. 30.

57. CC, déc. $\mathrm{n}^{\circ}$ 94-343/344 DC du 27 juillet 1994, Loi relative au respect du corps humain et loi relative au don et à l'utilisation des éléments et produits du corps humain, à l’assistance médicale à la procréation et au diagnostic prénatal, Rec., p. 10o, cons. 18.

58. Code pénal, art. 222-23. 
de lui fournir des locaux ou un véhicule afin qu'il puisse s'y livrer à la prostitution, sans même rechercher si la démarche du prostitué est volontaire. Il est pourtant difficile de comprendre en quoi celui qui est ici perçu comme un proxénète a asservi la personne prostituée si celle-ci a choisi d'exercer cette activité. La même problématique se pose à propos du délit de racolage. Lorsqu'un prostitué sollicite un individu en vue d'avoir des relations sexuelles tarifées avec lui, il ne saurait porter atteinte à la dignité de celui-ci; il peut éventuellement le choquer, mais il ne l'oblige aucunement à entretenir un rapport physique - et ce, encore moins en cas de racolage passif -, puisque l'intéressé peut refuser et passer son chemin. De même, si la personne prostituée racole volontairement, l'on ne comprend pas dans quelle mesure sa démarche est contraire au principe de dignité subjective tel qu'énoncé par le Conseil constitutionnel. La législation française, imprégnée de prohibitionnisme, est en réalité basée sur la conception renouvelée et objectivée du principe de dignité, puisqu'elle considère la prostitution consentie comme constitutive d'une atteinte à la dignité humaine.

Nul ne peut consentir à sa propre dégradation. L'inversion du concept traditionnellement subjectif de dignité fait surgir un «ordre public de protection individuelle ${ }^{59}$ qui tend à empêcher que les individus s'adonnent à des pratiques qui ne sont attentatoires qu'à leur propre intégrité physique, indépendamment de la protection d'autrui. L'exploitation commerciale d'un handicap physique est jugée indigne car elle viendrait nier la part d'humanité présente en chacun. Sur le fondement de la dignité objective et du principe d'égalité entre tous les hommes, il ne serait pas non plus possible de réifier son être et de consentir à se soumettre aux desiderata d'autrui. La prostitution apparaît comme une activité abjecte puisque, en échange d'une rémunération, un individu se transformerait en un objet de plaisir sexuel. Or, si la dignité est un principe matriciel duquel découlent l'ensemble des droits et libertés fondamentaux, $\mathrm{y}$ attenter revient à nier son statut d'homme libre. C'est pourquoi le système prohibitionniste juge indispensable d'interdire expressément le phénomène prostitutionnel dans sa globalité; la dignité s'offre ainsi comme un droit non renonçable. Nous sommes face à une dimension éminemment paternaliste de la dignité, qui aboutit à infantiliser les personnes prostituées. Ce sont les autorités publiques qui décident de ce qui représente un danger pour ces individus jugés vulnérables car incapables de s'en rendre compte par eux-mêmes.
Seule cette protection objective de la dignité permet de comprendre l'incrimination française des comportements racoleurs. En sollicitant un client potentiel, la personne prostituée considérerait son corps comme une simple marchandise à exploiter et ne lui témoignerait pas le respect qui lui est dû. En se chosifiant et en s'avilissant, elle contrevient ainsi à l'impératif catégorique kantien qui refuse l'instrumentalisation de l'être humain ${ }^{60}$. On note cependant une différence entre le système prohibitionniste et le système français. Le premier n'hésite pas à incriminer expressément la personne prostituée en raison de son activité indigne et à la condamner, tout comme l'individu qui va lui porter assistance. À l'inverse, le droit français rappelle in extremis le statut de victime des individus prostitués. Il refuse « de tirer jusqu'au bout les conséquences du véritable fondement des infractions » de proxénétisme de soutien ${ }^{61}$; ces délits sont fondés sur une conception objective de la dignité du prostitué, mais le législateur permet la seule pénalisation du proxénète en s'appuyant sur la protection de la dignité subjective de la victime. Le proxénète, en permettant à un individu de se prostituer - individu fragile qui ne perçoit pas l'indignité de son geste -, lui causerait un dommage.

Cette nuance exceptée, la logique prohibitionniste est fermement ancrée dans la législation nationale. En imposant péremptoirement la dignité aux personnes désirant monnayer leurs faveurs, les autorités nient finalement la capacité d'autodétermination de ces individus et affirment le principe d'indisponibilité du corps humain.

\section{Le principe d'indisponibilité du corps humain opposé aux prostitués}

«Je veux qu'il me batte, moi [...]. Il me plaît d'être battue ${ }^{62}$. Cette phrase célèbre de Martine revendiquant son libre arbitre et la libre disposition de son corps n'a plus lieu d'être dans les États qui prônent l'absolutisme de la dignité de la personne humaine. Le contentieux de la prostitution volontaire nous porte vers une question centrale: le corps et l'homme sont-ils indissociables ou l'homme, c'est-à-dire l'esprit, la volonté, domine-t-il son corps en exerçant sur lui un droit de propriété?

La législation actuelle semble pencher vers un principe d'indisponibilité du corps humain ou, du moins, ne reconnaît que ponctuellement à l'individu une libre disposition de son être. Ainsi, le droit autorise le don d'organes ou de sang, mais à condition que ces actes soient gratuits ${ }^{63}$.

59. Conclusions du commissaire du gouvernement M. Heers, sur les arrêts $M^{\text {me }}$ Donyoh et $M^{\text {me }}$ Senanayake rendus par la CAA de Paris le 9 juin 1998, Revue française de droit administratif, 1998, p. 1231-1240, à propos de la réalisation forcée de transfusion sanguine sur des patients adeptes du mouvement des Témoins de Jéhovah en cas d'urgence vitale.

60. "Agis de telle sorte que tu traites l'humanité aussi bien dans ta personne que dans la personne de tout autre, toujours en même temps comme une fin et jamais simplement comme un moyen» (E. Kant, Grundlegung zur Metaphysik der Sitten [Fondement de la métaphysique des mours], $2^{\mathrm{e}}$ édition, Riga, Hartkoch, 1786 , p. 81 .

61. J. Danet, «Atteinte à la dignité, pénalisation et prostitution », in Action publique et Prostitution, p. 61-62.

62. Molière, Le Médecin malgré lui [1667], acte I, scène II.

63. Code de la santé publique, art. L. 1211-1 sq. Code civil, art. 16-1, alinéa 3, 16-5, 16-6, 16-7. 
La non-patrimonialité du corps affirmée par l'assemblée plénière de la Cour de cassation dans un arrêt en date du 31 mai $1991^{64}$ à propos de l'illicéité d'une convention de mère porteuse apparaît symptomatique de l'absence de droit de propriété de l'homme sur son corps. L'article 1128 du Code civil rappelle qu' «il n'y a que les choses qui sont dans le commerce qui puissent être l'objet des conventions » et doit se lire à la lumière de l'article 16-1, alinéa 3, dudit Code qui pose l'absence de droit de patrimonialité sur le corps humain ainsi que sur ses éléments - un organe ou un membre - et sur ses produits - le sang, les gamètes. Il est ainsi interdit en France de «louer» un utérus ou d'acheter des ovules ou du sperme pour répondre à un désir parental. Cependant, l'application de ce principe de non-patrimonialité du corps et par suite de son indisponibilité semble malaisée à propos de la prostitution. En effet, la personne qui se prostitue ne se démembre pas ou ne donne pas en tant que telle une partie de son anatomie au client. La pratique sexuelle tarifée ne s'analyse pas systématiquement en un rapport sexuel; elle peut également être caractérisée par des caresses intimes, fellations ou actes de masturbation. Dans ces hypothèses, est-ce le corps ou un service de nature sexuelle qui est monnayé? Devrait-on également prohiber la réalisation de massages dans les salons d'esthétique au motif que son auteur, en utilisant ses mains, procure plaisir et bien-être à l'intéressé?

En raison de ce doute, le principe de non-patrimonialité du corps ne permet pas à lui seul de justifier la prohibition de la prostitution. La protection objective de la dignité se fonde sur l'indissociabilité du corps et de la personne; le corps n'est que le substrat de la personne, l'expression physique et matérielle de la personnalité. De ce fait, l'individu prostitué ne permettrait pas seulement au client de s'immiscer dans son intimité physique, mais il l'autoriserait à l'aliéner entièrement. Le client s'emparerait nécessairement du corps et de l'esprit du prostitué qui ne serait désormais plus l'auteur d'un acte sexuel, mais l'instrument, l'outil ${ }^{65}$. En se livrant volontairement à cette activité, la personne prostituée deviendrait l'esclave d'autrui.

La problématique de la prostitution volontaire révèle l'impossible neutralité éthique de l'État. L'engagement abolitionniste est fondé sur une ambiguïté extrême: il faut tolérer les échanges économico-sexuels non pas parce qu'ils relèvent de la liberté personnelle des individus s'y prêtant, mais parce que précisément, il ne faut pas accabler ces personnes victimes de leur condition. La prosti- tution est donc considérée comme un mal à proscrire par le biais de politiques sociales adéquates. Un antagonisme apparaît entre la législation réglementariste, qui reconnaît explicitement une liberté de disposer de son corps et d'en jouir financièrement, et les philosophies prohibitionniste et abolitionniste qui font primer la dignité objective sur la liberté personnelle. Ces dernières nient la réalité du consentement des personnes prostituées. «Quoi qu'on en dise [...], ce seront toujours les personnes démunies qui vendront leur rein ou leur foie, ou qui accepteront de porter un enfant pour une autre [...]. Seuls ceux ayant un pressant besoin d'argent "consentent" à travailler dans des conditions misérables, ou encore "consentent" à se prostituer. Ce sont les faibles qui consentent ${ }^{66}$. Le principe d'indisponibilité du corps humain permettrait au final de sauver des individus désespérés, en leur interdisant d'aggraver encore davantage leur situation.

L'éternel combat entre dignité "actuée» et dignité objective nous entraîne vers un domaine à la lisière du juridique, mêlant philosophie et sociologie: faut-il mieux reconnaître à l'individu le droit de jouir financièrement de son corps afin de pouvoir bénéficier de conditions de vie matérielles dignes, ou lui interdire l'accès à un tel «revenu» en arguant du fait qu'on ne peut vivre en niant sa part d'humanité? D'un côté, l'affirmation d'un droit de disposer de son corps engendre nécessairement un phénomène de marchandisation de l'être humain, qui ne pourra être borné que par l'atteinte aux droits ou libertés d'autrui. De l'autre, la négation totale du libre arbitre des personnes prostituées aboutit à la dangereuse instauration d'un ordre moral ${ }^{67}$, puisque l'invocation de la dignité apparaît floue et marquée d'un certain subjectivisme liberticide. En l'absence de critères établis et constitutifs de la dignité, il revient aux autorités compétentes de décider de ce qui est essentiel au respect de la nature humaine. La prostitution n'est pas interdite parce qu'elle soulève une réprobation nationale ou en raison de circonstances locales particulières, mais parce que le législateur prohibitionniste est intimement convaincu qu'un tel phénomène porte atteinte à l'humanité des individus s'y livrant. On pourrait ainsi dériver vers une société dans laquelle les autorités publiques imposent à la majorité leurs propres conceptions et valeurs morales ${ }^{68}$.

C'est en raison de ce risque que le Conseil d'État, dans son avis relatif à l'interdiction du port du voile intégral de mars $2010^{69}$, refuse de faire primer de façon absolue

64. Cour cass., assemblée plénière, 31 mai 1991, Procureur général de la Cour de cassation, Bull. civ., nº 4, Dalloz, 1991, Jur., p. 417 sq., rapport Y. Chartier, note D. Thouvenin; Semaine juridique, édition générale, 1991, II, 21782, communication J. Bernard, concl. H. Dontenwille, note F. Terré.

65. Sur ce point, voir l'analyse de B. Lavaud-Legendre, «Le droit pénal, la morale et la prostitution: des liaisons dangereuses », Droits. Revue française de théorie, de philosophie et de culture juridique, $\mathrm{n}^{\circ} 49,2009$, p. 57-81.

66. M. Fabre-Magnan, «Le domaine de l'autonomie personnelle: indisponibilité du corps humain et justice sociale», Recueil Dalloz, $\mathrm{n}^{\circ} 1,2008$, p. 35 .

67. Sur ce point, voir M. Canedo-Paris, «La dignité humaine en tant que composante de l'ordre public: l'inattendu retour en droit administratif français d'un concept controversé», Revue française de droit administratif, 2008, p. 996.

68. Selon G. Lebreton, «on parle d'ordre moral lorsque les autorités de police (et les juges) utilisent leurs prérogatives non plus pour faire respecter la moralité publique, mais pour imposer à la société leurs propres conceptions morales. Alors que la moralité publique vient d'en bas et constitue un facteur de paix sociale, l'ordre moral vient d'en haut et se veut un moyen de conquérir les âmes et d'opprimer les consciences» (G. Lebreton, "Ordre public et dignité de la personne humaine: un problème de frontière", in L'ordre public: Ordre public ou ordres publics? Ordre public et droits fondamentaux, M.-J. Redor (dir.), Bruxelles, Bruylant, 2001, p. 364).

69. Conseil d'État, «Étude relative aux possibilités juridiques d'interdiction du port du voile intégral», rapport adopté par l’assemblée générale plénière du Conseil d'État le jeudi 25 mars 2010, p. 17-21. Dans leur décision n ${ }^{\circ}$ 2010-613 DC du 7 octobre 2010, Loi interdisant la dissimulation du visage 
le principe de dignité sur la liberté personnelle des intéressées. Il relève qu' «il apparaît difficile de fonder un régime d'interdiction sur un fondement susceptible d'acceptions aussi variées et marqué, dans la pratique, d'une inévitable subjectivité», et affirme que «le fondement de la sauvegarde de la dignité est donc discutable juridiquement $[. .$.$] en particulier dans le cas où le port du voile$ intégral résulte de la volonté délibérée d'une personne majeure». Les conseillers d'État optent pour une approche plus subtile permettant de concilier ponctuellement les nécessités de l'ordre public et la liberté personnelle des femmes portant la burqa. Si l'on applique ce raisonnement au contentieux de la prostitution volontaire, le système prohibitionniste, atteint en son cœur, s'écroule. La position du juge administratif rejoint celle de l'assemblée parlementaire du Conseil de l'Europe qui avait indiqué en 2007 que «fonder son jugement sur le respect de la dignité humaine ne signifie pas pour autant adopter une démarche moraliste, mais plutôt respecter les décisions et les choix de chacun tant qu'ils ne nuisent pas à autrui ${ }^{70}$.

L'instauration d'un ordre moral est d'autant plus dangereuse qu'elle n'aboutit pas nécessairement à la protection effective de l'humanité des personnes prostituées. Si la prostitution est une activité indigne, pourquoi seul le racolage public est-il interdit? La sollicitation d'un individu en vue d'une relation sexuelle tarifée apparaît tout autant attentatoire à la dignité qu'elle ait lieu dans un espace public ou privé. La volonté d'éradiquer le phénomène prostitutionnel s'est diluée dans un combat contre la visibilité de cette activité. Il s'agit finalement davantage de protéger la société d'une activité considérée comme immorale que de lutter contre la réification des prostitués. Ce constat explique sans nul doute les nombreuses déficiences de la législation actuelle.

\section{B. Les déficiences d'une législation attentatoire aux droits des prostitués}

La législation française, qu'on la considère abolitionniste ou néo-prohibitionniste, comporte incontestablement des incohérences et des lacunes, préjudiciables au respect des droits des individus prostitués.

Si l'échange économico-sexuel tarifé est contraire à l'humanité des personnes s'y prêtant, il doit être interdit en tant que tel. La France doit dénoncer la Convention abolitionniste de 1949 et inscrire dans le droit pénal l'infraction de prostitution, afin que soit sauvegardé effectivement le principe de dignité. Se produirait alors un changement majeur : la criminalisation de l'ensemble des acteurs de la relation vénale, notamment celle des clients qui participent à la réification des prostitués. La lettre de l'article 225-10-1 du Code pénal porte à croire que l'auteur du délit de racolage peut être aussi bien une personne se livrant à la prostitution qu'un individu désirant payer pour avoir des relations sexuelles. Cependant, la question du client a toujours été exclue des débats parlementaires ${ }^{71}$, centrés uniquement sur l'instrumentalisation des personnes prostituées comme moyen de lutter contre les réseaux de proxénétisme. L'incitation à des pratiques charnelles en échange d'une rémunération ne se comprend que comme la sollicitation de la part d'un individu monnayant ses faveurs. L'infraction de racolage public n'est pas applicable à une personne non prostituée, sauf si le racolage est exercé sur un prostitué dont la minorité est connue ${ }^{72}$ ou sur une personne dont la vulnérabilité physique ou psychique est apparente ${ }^{73}$. En dehors de ces hypothèses limitatives, la responsabilité du client n'est pas retenue, même s'il vient solliciter les services d'une personne prostituée ${ }^{74}$.

L'absence de frein à la liberté d'action des clients apparaît déconcertante puisque les «consommateurs» qu'ils sont sont en partie responsables de l'existence d'une offre marchande de services sexuels. Comme tels, ils participent à l'économie souterraine des réseaux de proxénétisme et de traite des êtres humains. Il ne serait donc pas illogique que leur incrimination sur le chef de racolage soit envisagée au même titre que celle des personnes prostituées. La différence de traitement entre ces deux acteurs de la relation prostitutionnelle s'apparente à une violation du principe constitutionnel d'égalité devant la loi fondé sur l'article 6 de la Déclaration de $1789^{75}$. Pour la commission d'un même fait, le client est ignoré par le droit, le prostitué placé en garde à vue et traité comme l'auteur d'une infraction pénale. Le législateur justifie l'incrimination de racolage public par la nécessité de protéger les prostitués des réseaux de proxénétisme; le placement en garde à vue de la personne racoleuse permettrait à celle-ci de dénoncer son «mac» et d'aider à son arrestation. Même

dans l'espace public, les juges de la rue de Montpensier ne suivent pas le raisonnement du Conseil d'État et relèvent que l'action du législateur est nécessaire car la dissimulation du visage «peu[t] constituer un danger pour la sécurité publique et méconnaî[t] les exigences minimales de la vie en société» (cons. 4). L'évolution de l'ordre public vers l'inclusion de ces «exigences minimales» nous semble révélatrice du risque d'instauration d'un certain ordre moral. Que sont ces exigences? Qui en décide?

70. Assemblée parlementaire du Conseil de l'Europe, Prostitution - Quelle attitude adopter?, $\$ 6$.

71. Du moins, jusqu’à très récemment (voir la conclusion du présent article)

72. Code pénal, art. 225-12-1, alinéa 1. Par dérogation aux règles de compétence territoriale, l'article 225-13 dudit Code prévoit la répression de l'infraction lorsque celle-ci est commise à l'étranger par un Français ou toute personne résidant habituellement en France.

73. Code pénal, art. 225-12-1, alinéa 2. «Le fait de solliciter, d'accepter ou d'obtenir» des relations sexuelles de la part d'un mineur ou d'une personne particulièrement vulnérable se livrant à la prostitution, même de façon occasionnelle, est puni de 3 ans d'emprisonnement et de 45 ooo euros d'amende.

74. Voir Cass. crim., 25 mai 2005, $\mathrm{n}^{\circ}$ 04-84.769, Droit pénal, 2005, comm. 138, obs. Véron. Les juges y affirment que le fait de se trouver légèrement vêtu la nuit dans un endroit connu pour la prostitution est insuffisant pour constituer le délit de racolage, d'autant "que c'est le client qui a pris l'initiative d'aborder» l'individu prostitué en vue d'avoir avec lui des relations tarifées. La démarche volontaire du client n'est pas répréhensible et n'est prise en compte que pour écarter la responsabilité pénale du prostitué

75. CC, déc. $n^{\circ}$ 73-51 DC du 27 décembre 1973, Loi de finances pour 1974, Rec., p. 25, cons. 2. 
si le client n'est pas en lien direct avec les proxénètes et que, sauf cas exceptionnel, il ne peut pas aider les forces de l'ordre à démanteler un réseau, il influe nécessairement sur l'existence d'une telle organisation criminelle. Si l'intérêt général poursuivi par le législateur est la lutte contre les réseaux de proxénétisme, il est difficile de comprendre pourquoi l'action du client n'est pas prise en considération, ne serait-ce qu'a minima. De même, dès lors que l'on considère le délit de racolage fondé sur l'impératif de protection de la dignité objective de la personne prostituée, on s'aperçoit que la situation des deux protagonistes est proche: le prostitué porterait atteinte à sa dignité en considérant son corps comme une simple marchandise et le client participerait à cette réification en acceptant ses avances ${ }^{76}$. Il en serait même l'élément déclencheur s'il sollicite volontairement les faveurs d'un individu. En proposant un échange économico-sexuel, il considérerait autrui comme un objet de pure jouissance. Bien que la démarche intellectuelle ne soit pas identique dans ces deux hypothèses, il reste qu'elles conduisent au même constat de négation de l'humanité des prostitués. La différence de traitement entre prostitué et client ne semble donc pas justifiée.

La législation actuelle n'aboutit finalement qu'à la criminalisation et à la stigmatisation des seules victimes du phénomène et ce, en violation complète de l'engagement abolitionniste. Le fichage des personnes auteurs de racolage - et donc, monnayant leurs faveurs - est contraire aux prescriptions de la Convention onusienne de 1949, puisque l'article 6 de ce texte dénonce l'enregistrement «des personnes qui se livrent ou sont soupçonnées de se livrer à la prostitution ». Si le Conseil constitutionnel a validé cette disposition pourtant critiquable, c'est sous la réserve expresse que les autorités prennent en compte dans le prononcé de la peine le fait que le prévenu ait agi sous la menace ou contrainte ${ }^{77}$. La personne racolant sous l'emprise d'un proxénète voit sa sanction diminuer à condition de démontrer ce lien de subordination. Il est pourtant étonnant que l'existence d'une menace «hiérarchique» soit seulement prise en considération - telle une simple circonstance atténuante -, puisque l'article 122-2 du Code pénal prévoit que, dans une telle hypothèse, le prévenu est irresponsable pénalement ${ }^{78}$.

La situation juridique des prostitués étrangers est encore plus précaire. Alors que les articles 17 à 19 de la Convention onusienne pour la répression de l'exploita- tion de la prostitution d'autrui prévoient spécialement leur protection et leur réhabilitation, ces individus sont éloignés du territoire français lorsqu'ils sont passibles de poursuites pénales en matière de proxénétisme et de racolage $\mathrm{e}^{79}$ ou lorsque leur activité « constitue une menace grave pour l'ordre public ${ }^{80}$. Des mesures ont certes été prises afin de favoriser la collaboration entre « repentis du sexe tarifé» et policiers, mais leur ineffectivité est patente. L'article L. 316-1 du Code de l'entrée et du séjour des étrangers et du droit d'asile (CESEDA) ${ }^{81}$ prévoit qu'une carte de séjour temporaire portant mention «vie privée et familiale» et ouvrant droit à l'exercice d'une activité professionnelle peut être délivrée à l'étranger qui porte plainte contre un individu qu'il accuse d'avoir commis à son encontre des faits de proxénétisme ou de traite des êtres humains; cette possibilité est également ouverte pour l'étranger qui accepte de témoigner dans une procédure de ce type. L'alinéa 2 dudit article informe que cette carte devient une carte de résident - valable 10 ans et renouvelable - si l'individu mis en cause est condamné définitivement. Cependant, cette disposition n'est pas aussi favorable à ces individus qu'elle le laisse espérer. À la lecture des débats parlementaires relatifs à la sécurité intérieure en 2003, le placement en garde à vue des personnes prostituées étrangères a une finalité précise; il ne s'agit pas tant de les condamner pour comportement racoleur que de «pouvoir leur proposer soit de leur retirer leur titre de séjour et les raccompagner à la frontière, soit de prolonger leur titre de séjour, voire de leur accorder une autorisation de travail, si elles choisissent de coopérer au démantèlement du réseau qui l'exploite ${ }^{82}$.

Apparemment très courtoise, cette collaboration entre OPJ et prostitué étranger est pourtant le lieu de chantage dénoncé par le milieu associatif. De plus, le problème de la langue ainsi que la crainte du proxénète, beaucoup plus impressionnant aux yeux des prostitués que les représentants des forces de l'ordre, ne sont pas à même de permettre cette coopération. L'éloignement du migrant semble donc quasi certain.

Dans son avis sur la traite des êtres humains et l'exploitation sexuelle de 2009, la Commission nationale consultative des Droits de l'homme recommande vivement de délivrer un titre de séjour provisoire à toute personne prostituée étrangère faisant l'objet d'une enquête de police ${ }^{83}$, notamment au regard du droit à un recours effectif découlant de l'article 16 de la Déclaration des

76. Tout comme celui qui aide, assiste ou tolère la prostitution d'autrui participe au phénomène de réification (voir supra)

77. CC, déc. LSI, cons. 63

78. «Nul n'est pénalement responsable s'il a agi sous la menace ou par contrainte».

79. CESEDA, art. 313-5

80. CESEDA, art. 521-1. La prostitution n'étant pas interdite, le seul fait de s'y livrer ne constitue pas une menace pour l'ordre public. Les mesures d'éloignement sont justifiées par les manifestations extérieures de la prostitution et les nuisances occasionnées. Voir CE, 16 mars $2005, \mathrm{n}^{\circ} 269313$; CAA Lyon, 9 juillet $2008, \mathrm{n}^{\circ}$ o8LYoo 411 et 08 LYoo 412.

81. Introduit par l'article 76 de la LSI du 18 mars 2003, modifié par l'article 39 de la loi nº $2006-911$ relative à l'immigration et à l'intégration du 24 juillet 2006, publiée au JORF du 25 juillet.

82. Assemblée nationale, $1^{\text {re }}$ et $2^{\mathrm{e}}$ séances publiques du 21 janvier 2003.

83. Avis de la Commission nationale consultative des Droits de l'homme adopté par l'assemblée plénière du 18 décembre 2009 , p. 15 , 20 et 26 (disponible sur http.//www.cncdh.fr/IMG/pdf/Avis_traite_et_l_exploitation_des_etres_humains_en_France.pdf). 
Droits de l'homme et du citoyen ${ }^{84}$. La Commission souligne également la nécessité de supprimer l'infraction de racolage public qui "occasionne un préjudice secondaire" aux victimes et qui s'oppose au devoir étatique de protection et de réhabilitation des prostitués posé à l'article 16 de la Convention de $1949^{85}$. En la matière, l'action sociale de l'État n'est que très théorique ${ }^{86}$. Un dispositif d'assistance aux « survivants » de la prostitution est prévu, notamment par l'article L. 121-9 du Code de l'action sociale et des familles, mais le faible nombre de places dans les centres d'hébergement et de réinsertion sociale rend la protection fictive. Les autorités publiques se défaussent progressivement de leur mission sur les différentes associations nationales, parmi lesquelles L'Amicale du Nid, SOS Femmes Accueil, Les amis du bus de femmes, qui dénoncent régulièrement le manque de moyens logistiques et financiers mis à leur disposition. En outre, la LSI de 2003 a abouti à la hausse de l'insécurité sanitaire et physique des prostitués en obligeant ceux-ci à s'éloigner des zones d'habitation pour ne pas risquer d'être réprimés pour racolage public. Par là, elle a complexifié le travail des différents secteurs associatifs et accentué la situation de précarité des individus concernés. Le désengagement de l'État apparaît donc entier, excepté peut-être dans le domaine fiscal, puisque les revenus prostitutionnels sont imposés.

Régulièrement critiqué, ce « réalisme fiscal ${ }^{87}$ semble se confondre avec les délits de proxénétisme de profit et de contrainte définis aux articles $225-2,2^{\circ}$ et $3^{\circ}$ du Code pénal. Saisie de ce contentieux, la CEDH a rendu le 11 septembre 2007 un arrêt Tremblay c. France pour le moins controversé $^{88}$. Ancienne prostituée désirant se reconvertir, la requérante sollicitait son affiliation auprès de l'Urssaf en qualité de décoratrice. Au terme d'une enquête, l'organisme lui réclamait environ 40000 euros de cotisations et majorations au titre de son ancienne activité. L'intéressée dénonçait l'entrave à sa réinsertion constituée par l'obligation qui lui était faite de s'acquitter de cette somme.
Pour combler sa dette, elle n'avait d'autre choix que de se prostituer à nouveau, ce qui «l'enferm[ait] dans un cercle vicieux» $(\$ 19)$ puisque les bénéfices générés donneraient lieu à de nouvelles impositions et cotisations. En empêchant son reclassement, les autorités publiques auraient violé les articles 3 et $4 \$ 2$ de la CEDH, en la soumettant à un traitement dégradant et en la contraignant à accomplir un travail forcé ou obligatoire.

La CEDH, extrêmement sévère, n'accueille pas les prétentions de l'intéressée. Elle relève que «la prostitution [est] incompatible avec les droits et la dignité de la personne humaine dès lors qu'elle est contrainte ${ }^{89}$, mais n'est pas convaincue que l'attitude de l'Urssaf s'analyse en une telle contrainte. L'obligation de payer les cotisations a certes «rendu malaisée la cessation de l'activité prostitutionnelle $[\ldots]$ et entravé son projet de réinsertion » $(\$ 33)$, mais l’organisme n’a jamais exigé expressément que la requérante s'acquittât de ses dettes en continuant à se prostituer et il a tenu compte de la situation difficile de l'intéressée en acceptant de procéder à l'échelonnement des versements. En refusant de reconnaître le proxénétisme d'État et la contrainte « de fait» due au harcèlement fiscal, les juges de Strasbourg font clairement primer l'intérêt économique de l'État sur son devoir de secours des individus prostitués « repentis». L'imposition des revenus prostitutionnels reste un problème épineux. Au regard de la collaboration entre l'administration fiscale et les services de police ${ }^{90}$, il est possible de soutenir que cette pratique permettrait de ne pas encourager l'exercice de la prostitution. À l'inverse, d'aucuns affirment que la fiscalisation aurait pour conséquence de reconnaître implicitement la prostitution comme une activité professionnelle générant des bénéfices imposables, à l'instar de tout travail, ce qui irait à l'encontre de la philosophie abolitionniste. Cependant, la remise en cause de l'assujettissement fiscal des prostitués ne pourrait se réaliser que par le biais d'une réglementation particulière qui serait, elle aussi, contraire à la logique abolitionniste.

84. CC, déc. nº 96-373 DC du 9 avril 1996, Loi organique portant statut d'autonomie de la Polynésie française, Rec., p. 43 , cons. 83.

85. Cet article dispose que «les Parties à la présente Convention conviennent de prendre ou d'encourager, par l'intermédiaire de leurs services sociaux, économiques, d'enseignement, d'hygiène et autres services connexes, qu'ils soient publics ou privés, les mesures propres à prévenir la prostitution et à assurer la rééducation et le reclassement des victimes de la prostitution et des infractions visées par la présente Convention". L'on retrouve le même dispositif à l'article 11.3.1 de la Convention du Conseil de l'Europe de 2005 relative à la traite des êtres humains précitée.

86. Il nous semble que la déficience des autorités nationales dans la mise en œuvre de leur devoir de réhabilitation des personnes prostituées contrevient et à la pensée abolitionniste et à la philosophie prohibitionniste. Dans cette seconde hypothèse, l'action sociale de l'État devrait être renforcée si l'exercice de la prostitution viole effectivement le principe de dignité de la personne humaine. En plus de s'abstenir d'empêcher la réhabilitation des repentis de la prostitution, les États prohibitionnistes auraient l'obligation de la favoriser par la mise en place de campagnes publicitaires adéquates ou encore par l'octroi de subventions aux associations afin de leur donner les moyens d'agir efficacement.

87. D. Derycke, Les politiques publiques et la prostitution..., p. 73-78 (voir précisément p. 78 in fine).

88. $\mathrm{CEDH}, 2^{\mathrm{e}}$ section, 11 septembre 2007 , req. $\mathrm{n}^{\mathrm{o}} 37194 / 02$, Tremblay $c$. France. Pour un exposé détaillé de la situation fiscale des prostitués en France, voir G. François-Dainville, «La prostitution et le droit de la Sécurité sociale: la question de l'affiliation», Droit social, 2005, p. 888-892; C. Geslot, "Prostitution, dignité... Par ici la monnaie! », Recueil Dalloz, nº 19, 2008, p. 1292-1299; J.-M. Larralde, "La France, État proxénète? », Revue trimestrielle des Droits de l'homme, $\mathrm{n}^{\circ}$ 77, 2009, p. 195-210.

89. \$25 de l'arrêt. Les juges ne qualifient en revanche pas la prostitution volontaire au regard de l'article 3 , puisque la requérante ne soutient pas que la prostitution est en elle-même constitutive d'un traitement inhumain ou dégradant.

90. Sur le fondement de l'article 40 du Code de procédure pénale, les services de police ont l'obligation d'informer le procureur de la République de l'existence de tout crime ou délit; le déclenchement de poursuites pour proxénétisme ou pour racolage est de nature à inciter les services fiscaux à vérifier l'état des finances des prévenus. De même, l'article L. 101 du Livre des procédures fiscales dispose que l'autorité judiciaire est tenue de communiquer à l'administration des finances toute indication de nature à faire présumer une fraude. 
On aurait pu espérer que le rapport de la Mission d'information de l'Assemblée nationale sur la prostitution en France ${ }^{91}$ soulève les nombreux dysfonctionnements et inadaptations de la législation en vigueur et y apporte des éléments de réponse. Mais bien que ce document soit complet, fourni en chiffres et en témoignages d'individus prostitués, de clients, d'associations ou encore de politiques, il apparaît décevant en ce qu'il fait un amalgame entre prostitution forcée et prostitution consentie. Surtout, les trente propositions du rapport ne permettent toujours pas de trancher entre abolitionnisme et prohibitionnisme. D’un côté, le rappel de la nécessaire prévention du phénomène prostitutionnel ainsi que de la protection et de la réhabilitation effectives des prostitués le demandant fait écho à la philosophie abolitionniste traditionnelle ${ }^{92}$. De l'autre, la proposition de maintien du délit de racolage public actif et passif et celle de création d'un délit sanctionnant le recours à la prostitution

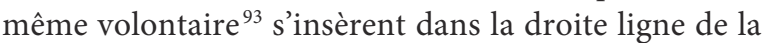
pensée prohibitionniste, au risque d'institutionnaliser le primat de la dignité objective - notion dangereusement incertaine - et au détriment de la liberté personnelle de chacun.

91. Rapport $\mathrm{n}^{\circ} 3334$ de la Mission d'information sur la prostitution en France, «Prostitution, l'exigence de responsabilité: en finir avec le mythe du plus vieux métier du monde», enregistré à la présidence de l'Assemblée nationale le 13 avril 2011. Ce rapport a été rendu après la rédaction de cet article, ce qui explique que seule cette rapide conclusion y fasse référence et que les mesures préconisées, bien qu'intéressantes, ne soient pas davantage analysées.

92. La moitié des propositions de la Mission concernent la nécessité d'améliorer la situation des prostitués et notamment leur réinsertion sociale, en leur accordant des remises fiscales gracieuses, un revenu de substitution, des possibilités accrues d'hébergement... (voir p. 311-315).

93. La pénalisation de l'achat de services sexuels, première proposition du rapport, rejoint directement la pensée prohibitionniste: puisque la prostitution est considérée comme une activité indigne, elle doit être interdite, et tout individu participant ou sollicitant la réification sexuelle d'une personne doit être empêché. Il n'est cependant pas certain que la proposition aboutisse à la création d'un délit; il s'agirait davantage de responsabiliser les potentiels clients, de leur faire comprendre la gravité de leur geste par le biais d'un simple rappel à la loi. 